\title{
Metformin suppresses intrahepatic coagulation activation in mice with lipopolysaccharide/D-galactosamine-induced fulminant hepatitis
}

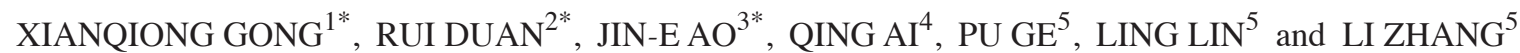 \\ ${ }^{1}$ Department of Liver Diseases, Hepatology Center, Xiamen Hospital of Traditional Chinese Medicine, Xiamen, Fujian 361000; \\ Departments of ${ }^{2}$ General Surgery and ${ }^{3}$ Pathology, The First People's Hospital of Jingmen, Jingmen, Hubei 448000; \\ Departments of ${ }^{4}$ Physiology and ${ }^{5}$ Pathophysiology, Chongqing Medical University, Chongqing 400016, P.R. China
}

Received November 4, 2014; Accepted July 28, 2015

DOI: $10.3892 / \mathrm{mmr} .2015 .4206$

\begin{abstract}
Metformin is a widely-used antidiabetic drug with hypoglycemic activity and previously described anti-inflammatory properties. Previous studies have demonstrated that metformin attenuates endotoxic hepatitis, however the mechanisms remain unclear. Inflammation and coagulation are closely associated pathological processes, therefore the potential effects of metformin on key steps in activation of the coagulation system were further investigated in endotoxic hepatitis induced by lipopolysaccharide/D-galactosamine (LPS/D-Gal). The current study demonstrated that treatment with metformin significantly suppressed the upregulation of tissue factor and plasminogen activator inhibitor-1 in LPS/D-Gal-exposed mice. In addition, a reduction in the expression of interleukin 6 and inhibition of nuclear translocation of nuclear factor $-\kappa \mathrm{B}$ were observed. These data indicate that the LPS/D-Gal-induced elevation of the stable protein level of hypoxia inducible factor $1 \alpha$, the mRNA level of erythropoietin, vascular endothelial growth factor and matrix metalloproteinase-3, and the hepatic level of lactic acid were also suppressed by metformin. The current study indicates that the suppressive effects of metformin on inflammation-induced
\end{abstract}

Correspondence to: Professor Li Zhang, Department of Pathophysiology, Chongqing Medical University, 1 Yixueyuan Road, Chongqing 400016, P.R. China

E-mail: zhangli@cqmu.edu.cn

*Contributed equally

Abbreviations: D-Gal, D-galactosamine; EPO, erythropoietin; HIF-1 $\alpha$, hypoxia inducible factor $1 \alpha$; IL-6, interleukin 6; IкB, inhibitory $\kappa \mathrm{B}$; LA, lactic acid; LPS, lipopolysaccharide; MMP-3, matrix metalloproteinase-3; NF- $\kappa \mathrm{B}$, nuclear factor $\kappa \mathrm{B}$; PAI-1, plasminogen activator inhibitor-1; TF, tissue factor; TNF- $\alpha$, tumor necrosis factor $\alpha$; VEGF, vascular endothelial growth factor

Key words: lipopolysaccharide, metformin, coagulation, tissue factor, hepatitis coagulation may be an additional mechanism underlying the hepatoprotective effects of metformin in mice with LPS/D-Gal-induced fulminant hepatitis.

\section{Introduction}

Fulminant hepatitis has been reported to result from a variety of causes, such as viral infection and drug poisoning, and is commonly associated with a poor prognosis $(1,2)$. In the early stages of fulminant hepatitis, the excessive inflammatory reactions serve a critical role in mediating the rapid loss of hepatocytes $(3,4)$. Therapies targeting the pro-inflammatory mediators are suggested to provide protective benefits in experimental studies $(5,6)$. In addition to the direct detrimental effects of pro-inflammatory mediators, inflammation may also activate the coagulation system $(7,8)$. The inflammation-induced activation of coagulation cascades markedly potentiates liver damage via interrupting the blood supply and propagating further inflammation $(9,10)$.

It has been well documented that inflammation and inflammation-induced coagulation are crucial pathological mechanisms underling the progression of critical disorders, including sepsis and multiple organ dysfunction syndrome $(11,12)$. The rapid induction of tissue factor (TF) under inflammatory circumstances is the pivotal step associating inflammation with coagulation (13). TF is the essential initiator of the extrinsic pathway of blood coagulation (14). The inflammatory stimuli and inflammatory cytokines may markedly induce the expression of TF and activate the extrinsic coagulation pathway (15). In addition to enhanced activation of coagulation cascades, inflammation may induce the dysregulation of fibrin removal via the upregulation of the plasminogen activator inhibitor-1 (PAI-1) $(16,17)$.

A previous study demonstrated that the antidiabetic drug, metformin, attenuated lipopolysaccharide/D-galactosamine (LPS/D-Gal)-induced fulminant hepatitis (18). LPS/D-Gal-induced fulminant liver damage predominantly depends on the induction of the pro-inflammatory cytokine, tumor necrosis factor $\alpha$ (TNF- $\alpha$ ), which has been proposed as a key factor propagating the progression of hepatitis induced by toxic insults $(4,19)$. In a previous study, metformin significantly 
suppressed the expression of TNF- $\alpha$, alleviating the liver lesions and improving the survival rate of LPS/D-Gal-exposed mice (18).

Due to evidence indicating that the coagulation system is involved in the pathogenesis of LPS/D-Gal-induced fulminant hepatitis $(20,21)$, the current study investigated whether metformin is able to modulate the activation of the coagulation response. In addition, the potential effects of metformin on LPS/D-Gal-induced upregulation of TF and PAI-1 and the subsequent metabolic disturbances were investigated.

\section{Materials and methods}

Materials. Metformin, LPS (from Escherichia coli, 055:B5) and D-Gal were purchased from Sigma-Aldrich (St. Louis, MO, USA). The lactic acid (LA) assay kit was purchased from Nanjing Jiancheng Bioengineering Institute (Nanjing, China). The Total Protein Extraction kit was purchased from Beyotime Institute of Biotechnology (Jiangsu, China). The mouse interleukin 6 (IL-6) Enzyme-Linked Immunosorbent Assay (ELISA) kit was obtained from NeoBioscience Technology Company (Shenzhen, China). The rabbit anti-mouse monoclonal nuclear factor $\kappa \mathrm{B}(\mathrm{NF}-\kappa \mathrm{B})$ p65 antibody (D14E12; 1:1,000; cat. no. 8242), rabbit anti-mouse hypoxia inducible factor $1 \alpha(\mathrm{HIF}-1 \alpha)$ monoclonal antibody (D2U3T; 1:1,000; cat. no. 14179), rabbit anti-mouse PAI-1 monoclonal antibody (D9C4; 1:1,000; cat. no. 11907), rabbit anti-mouse $\beta$-actin monoclonal antibody (D6A8; 1:1,000; cat. no. 8457) and Histone 3: rabbit anti-mouse Histone 3 monoclonal antibody (D1H2; 1:500; cat. no. 4499) were purchased from Cell Signaling Technology, Inc. (Danvers, MA, USA). The rabbit anti-mouse TF monoclonal antibody (EPR8986; 1:1,000; cat. no. ab151748) was purchased from Abcam (Cambridge, $\mathrm{UK})$. The bicinchoninic acid (BCA) protein assay kit, horseradish peroxidase-conjugated goat anti-rabbit polyclonal secondary antibody (1:5,000; cat. no. G21234) and enhanced chemiluminescence (ECL) reagents were obtained from Pierce Biotechnology, Inc. (Rockford, IL, USA).

Animals. A total of 64 male Balb/c mice (16 in each group), weighing 20-25 g, were obtained from the Experimental Animal Center of Chongqing Medical University (Chongquing, China). All animals were fed with a standard laboratory diet and water ad libitum. They were housed in a specific pathogen-free room at a temperature of $20-25^{\circ} \mathrm{C}$ and $50 \pm 5 \%$ relative humidity under a $12 \mathrm{~h}$ dark/light cycle and had acclimatized for a minimum of 1 week prior to use. All experimental procedures involving animals were approved by the Animal Care and Use Committee of Chongqing Medical University.

Induction of fulminant hepatitis. Balb/c mice were intraperitoneally injected with vehicle or metformin (400 mg/kg, dissolved in normal saline), administered $0.5 \mathrm{~h}$ prior to the injection of LPS (10 $\mu \mathrm{g} / \mathrm{kg})$ combined with D-Gal (700 $\mathrm{mg} / \mathrm{kg}$ ). The dose of metformin was selected based on previous experimental data (18). Subsequent to injection, the animals were returned to their cages and allowed food and water ad libitum. The experimental animals were allocated to four groups: Control $(\mathrm{CON})$, mice received vehicle administration only; metformin (MET), mice treated with metformin without LPS/D-Gal exposure; LPS/D-Gal, mice exposed to LPS/D-Gal; LPS/D-Gal + MET, mice treated with metformin and exposed to LPS/D-Gal. Mice were sacrificed by intraperitoneal injection of pentobarbital

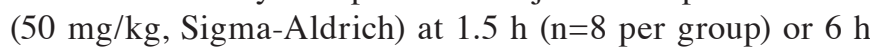
( $n=8$ per group) subsequent to LPS/D-Gal challenge, and blood samples and liver tissues were harvested and stored at $-80^{\circ} \mathrm{C}$ until required.

Reverse transcription-quantitative polymerase chain reaction $(R T-q P C R)$. Total RNA was isolated from liver samples using TRIzol reagent (Takara Bio, Inc., Otsu, Japan). First-strand complementary DNA was synthesized from $1 \mu \mathrm{g}$ total RNA using oligo-dT primer (Takara Bio, Inc.) and the M-MLV reverse transcriptase (Takara Bio, Inc.). RT-qPCR was performed with SYBR green PCR Master Mix (Takara Bio, Inc.). The sequences of the primers used to amplify the target genes are presented in Table I. PCR was performed on a CFX96 Touch (Bio-Rad Laboratories, Inc., Hercules, CA, USA) using the following PCR conditions: Denaturing at $95^{\circ} \mathrm{C}$ for $10 \mathrm{sec}$, annealing at $58^{\circ} \mathrm{C}$ for $20 \mathrm{sec}$ and elongation at $72^{\circ} \mathrm{C}$ for $20 \mathrm{sec}$. The mRNA levels of TF, PAI-1, IL-6, erythropoietin (EPO), vascular endothelial growth factor (VEGF) and matrix metalloproteinase-3 (MMP-3) were normalized to levels of $\beta$-actin.

Western blot analysis. Total proteins from liver samples were prepared using the Total Protein Extraction kit according to the manufacturer's instructions. The total protein concentration was determined using the BCA protein assay kit. Protein extracts were fractionated on a $10 \%$ polyacrylamide-sodium dodecyl sulfate gel (Beyotime Institute of Biotechnology) and then were transferred to nitrocellulose membranes (Pierce Biotechnology, Inc.). The membranes were blocked with $5 \%(\mathrm{w} / \mathrm{v})$ nonfat milk in Tris-buffered saline containing 0.05\% Tween-20 (Enzo Life Sciences, Farmingdale, NY, USA), prior to incubation with primary antibodies overnight at $4^{\circ} \mathrm{C}$, followed by incubation with secondary antibodies for $1 \mathrm{~h}$ at $37^{\circ} \mathrm{C}$. Antibody binding was visualized using an ECL system and a short exposure of the membrane to X-ray films (Kodak, Rochester, NY, USA).

ELISA. The protein levels of IL-6 in the plasma were determined using the ELISA kits according to the manufacturer's instructions (NeoBioscience Technology Company).

LA measurement. The LA contents in the liver tissue were determined with an LA assay kit according to the manufacturer's instructions. The LA values were assessed according to the absorbance measured at $530 \mathrm{~nm}$ (Varioscan Flash; Thermo Fisher Scientific, Waltham, MA, USA). The levels of hepatic LA were normalized to the total protein concentration in the same sample.

Statistical analysis. All data were presented as the mean \pm standard deviation. Statistical significance was determined by Student's t-test for the comparison of two groups. Data were analyzed using Statistical Package for Social Sciences (SPSS) software (version 16; SPSS, Inc., Chicago, 
Table I. Sequences of the primers for reverse transcription-quantitative polymerase chain reaction.

\begin{tabular}{lll} 
Target gene & \multicolumn{1}{c}{ Forward primer } & \multicolumn{1}{c}{ Reverse primer } \\
\hline TF & 5-CTTATCGGAAAGGCTCAA-3 & 5-CACCACTGCTCCCACAAT-3 \\
PAI-1 & 5-CATGTTTAGTGCAACCCTGGC-3 & 5-TGAGATGACAAAGGCTGTGGAG-3 \\
IL-6 & 5-AGTTGCCTTCTTGGGACTGATG-3 & 5-TCTCATTTCCACGATTTCCCAG-3 \\
EPO & 5-CAGCCACCAGAGACCCTTCA-3 & 5-TGTGAGTGTTCGGAGTGGAGC-3 \\
VEGF & 5-ACGATGAAGCCCTGGAGTGC-3 & 5-GCTCATCTCTCCTATGTGCTGGC-3 \\
MMP-3 & 5-CCACAGACTTGTCCCGTTTCC-3 & 5-GTGCTGACTGCATCAAAGAACAA-3 \\
$\beta$-actin & 5-CTGAGAGGGAAATCGTGCGT-3 & 5-CCACAGGATTCCATACCCAAGA-3
\end{tabular}

TF, tissue factor; PAI-1, plasminogen activator inhibitor-1; IL-6, interleukin 6; EPO, erythropoietin; VEGF, vascular endothelial growth factor; MMP-3, matrix metalloproteinase-3.

A

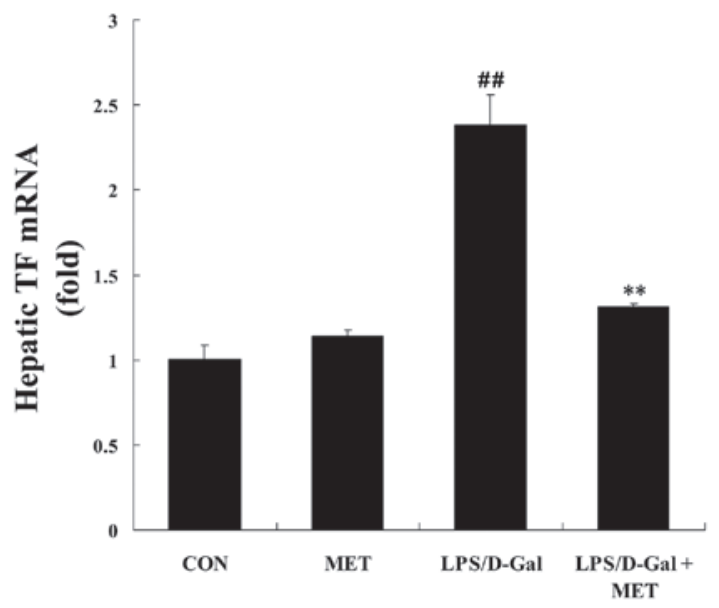

B

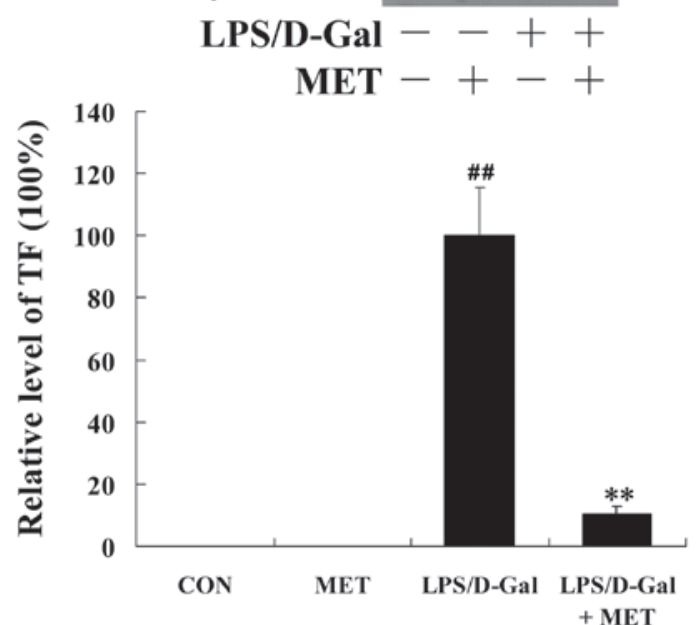

Figure 1. MET suppressed LPS/D-Gal-induced upregulation of TF. Mice were treated with the vehicle or MET (400 mg/kg) in the presence or absence of LPS/D-Gal. (A) The mRNA level of TF $1.5 \mathrm{~h}$ subsequent to LPS/D-Gal exposure and (B) the protein levels of TF $6 \mathrm{~h}$ subsequent to LPS/D-Gal exposure were determined. The bands of TF and $\beta$-actin are indicated by arrows. The blots underwent densitometric analysis and the data are presented as relative intensity units. Data are presented as the mean \pm standard deviation, $n=8 .{ }^{* *} \mathrm{P}<0.01$ vs. LPS/D-Gal group; ${ }^{\# \#} \mathrm{P}<0.01$, vs. CON group. MET, metformin; LPS, lipopolysaccharide; D-Gal, D-galactosamine; TF, tissue factor; CON, control.
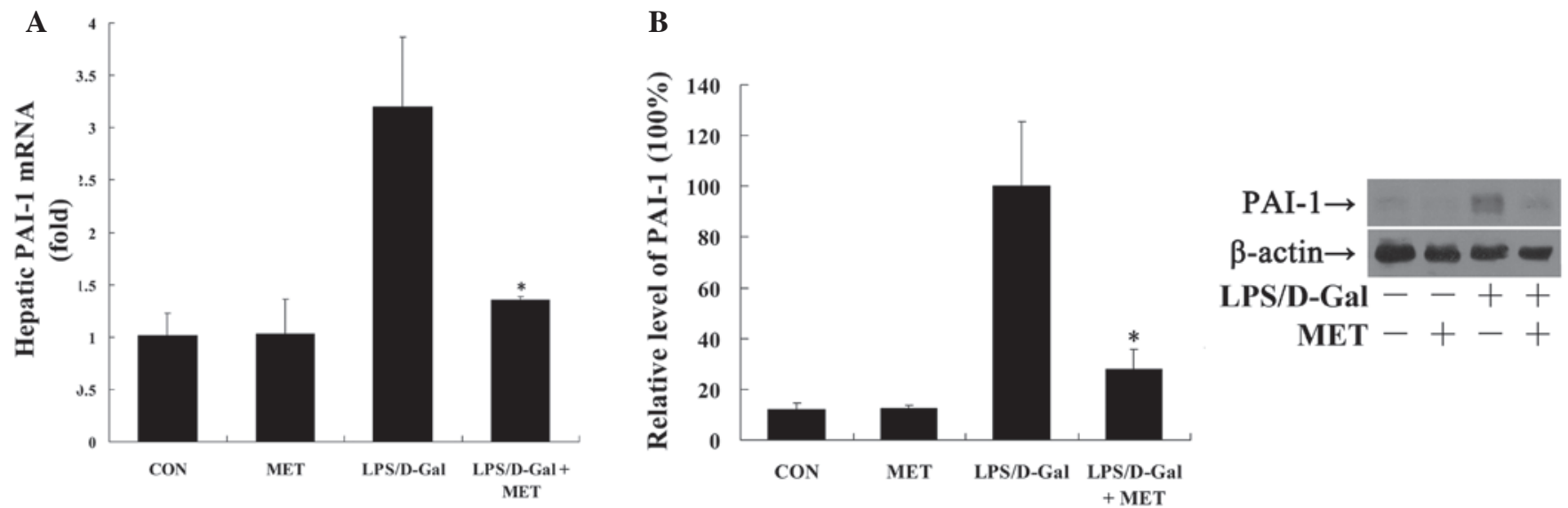

Figure 2. MET suppressed LPS/D-Gal-induced upregulation of PAI-1. Mice were treated with vehicle or MET (400 mg/kg) in the presence or absence of LPS/D-Gal. (A) The mRNA level of PAI-1 $1.5 \mathrm{~h}$ subsequent to LPS/D-Gal exposure and (B) the protein levels of PAI-1 6 h subsequent to LPS/D-Gal exposure were determined. The bands of PAI-1 and $\beta$-actin are indicated by arrows. The blots underwent densitometric analysis and the data are presented as relative intensity units. Data are presented as the mean \pm standard deviation, $n=8$. ${ }^{*} \mathrm{P}<0.05$ vs. LPS/D-Gal group. MET, metformin; LPS, lipopolysaccharide; D-Gal, D-galactosamine; PAI-1, plasminogen activator inhibitor type 1 ; CON, control. 

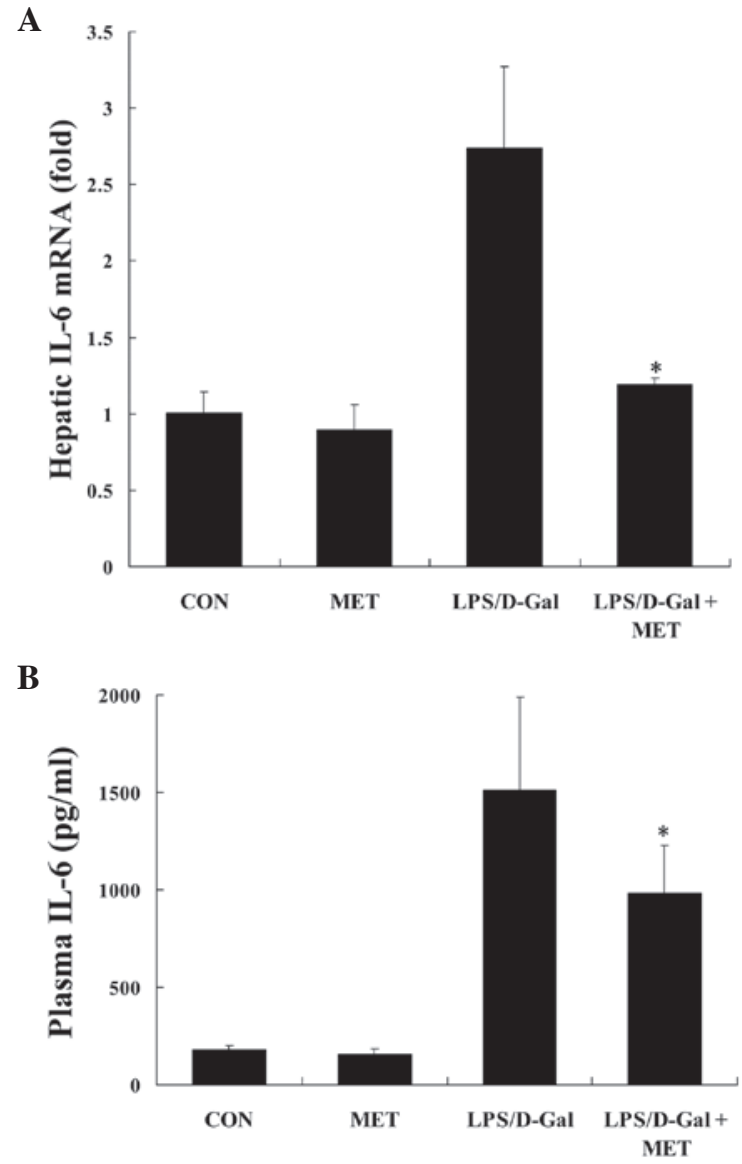

Figure 3. MET suppressed LPS/D-Gal-induced expression of IL-6. Mice were treated with vehicle or MET $(400 \mathrm{mg} / \mathrm{kg})$ in the presence or absence of LPS/D-Gal exposure. (A) The mRNA levels of IL-6 in the liver and (B) the protein levels of IL-6 in the plasma were determined $1.5 \mathrm{~h}$ subsequent to LPS/D-Gal exposure. Data are presented as the mean \pm standard deviation, $\mathrm{n}=8$. "P<0.05 vs. LPS/D-Gal group. MET, metformin; LPS, lipopolysaccharide; D-Gal, D-galactosamine; IL-6, interleukin 6; CON, control.

IL, USA). $\mathrm{P}<0.05$ was considered to indicate a statistically significant difference.

\section{Results}

Metformin suppresses LPS/D-Gal-induced TF and PAI-I expression. TF is the initiator of the coagulation cascade, which is rapidly induced by inflammatory stimuli (13). In the present study, LPS/D-Gal exposure was demonstrated to markedly upregulate the mRNA and protein levels of TF, compared with the control, which was significantly reversed by metformin $(\mathrm{P}<0.01 ;$ Fig. 1). In addition, the LPS/D-Gal-induced expression of PAI-1, an inflammation-induced factor suppressing the fibrinolysis process (17), was also significantly inhibited by metformin (P<0.05; Fig. 2).

Metformin suppresses LPS/D-Gal-induced IL-6 expression. IL-6 is a crucial pro-inflammatory cytokine linking inflammation to coagulation (22). In the current study, it was demonstrated that the challenge with LPS/D-Gal induced the upregulation the mRNA levels of IL- 6 in liver tissue, whereas treatment with metformin significantly reduced the level of IL-6 ( $\mathrm{P}<0.05$; Fig. 3A). In addition, similar inhibitory
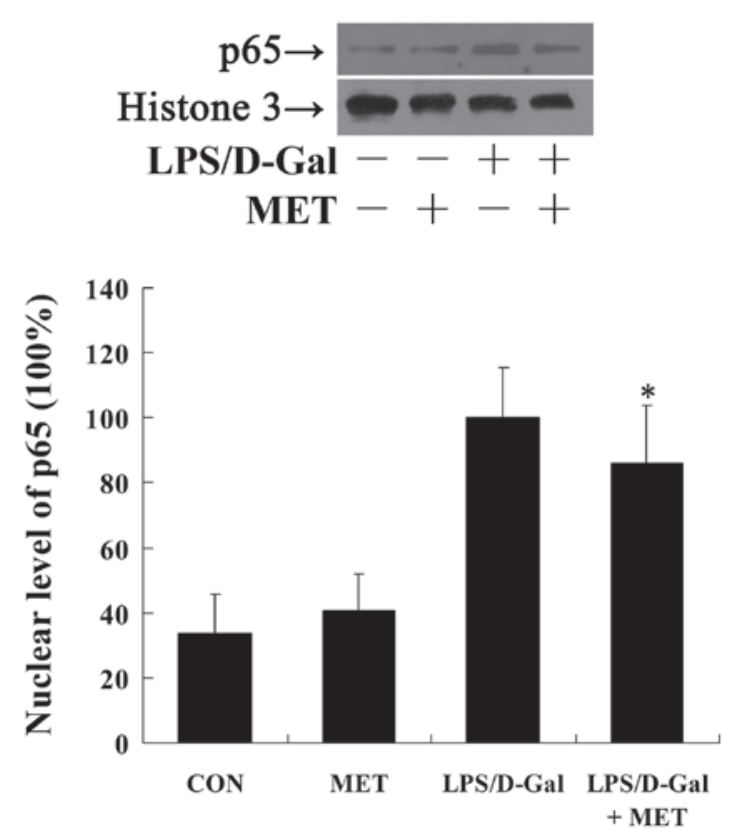

Figure 4. MET blocked LPS/D-Gal-induced nuclear translocation of NF- $\kappa B$ p65. Mice were treated with vehicle or MET $(400 \mathrm{mg} / \mathrm{kg})$ in the presence or absence of LPS/D-Gal. Liver samples were harvested $1.5 \mathrm{~h}$ subsequent to LPS/D-Gal exposure. The levels of p65 in nuclear proteins were determined by immunoblot analysis. The bands of p65 and histone 3 are indicated by arrows. The blots underwent densitometric analysis and the data are presented as relative intensity units. Data are presented as the mean \pm standard deviation, $n=8$. " $\mathrm{P}<0.05$, vs. LPS/D-Gal group. MET, metformin; LPS, lipo-

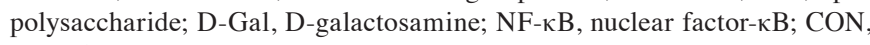
control.
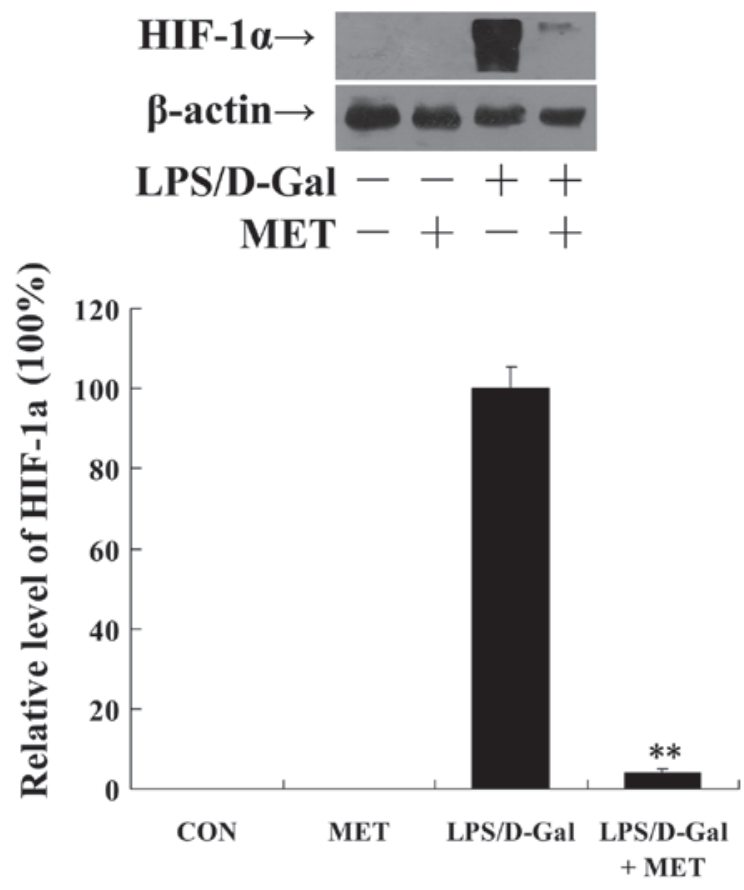

Figure 5. MET suppressed LPS/D-Gal-induced upregulation of HIF-1 $\alpha$. Mice were treated with vehicle or MET $(400 \mathrm{mg} / \mathrm{kg})$ in the presence or absence of LPS/D-Gal exposure. Liver samples were harvested $6 \mathrm{~h}$ subsequent to LPS/D-Gal exposure and the protein levels of HIF-1 $\alpha$ were determined. The bands of HIF- $1 \alpha$ and $\beta$-actin are indicated by arrows and the blots underwent densitometric analysis and the data are presented as relative intensity units. Data are presented as the mean \pm standard deviation, $\mathrm{n}=8$. ${ }^{* *} \mathrm{P}<0.01$ vs. LPS/D-Gal group. MET, metformin; LPS, lipopolysaccharide; D-Gal, D-galactosamine; HIF-1 $\alpha$, hypoxia inducible factor $1 \alpha$; CON, control. 
A

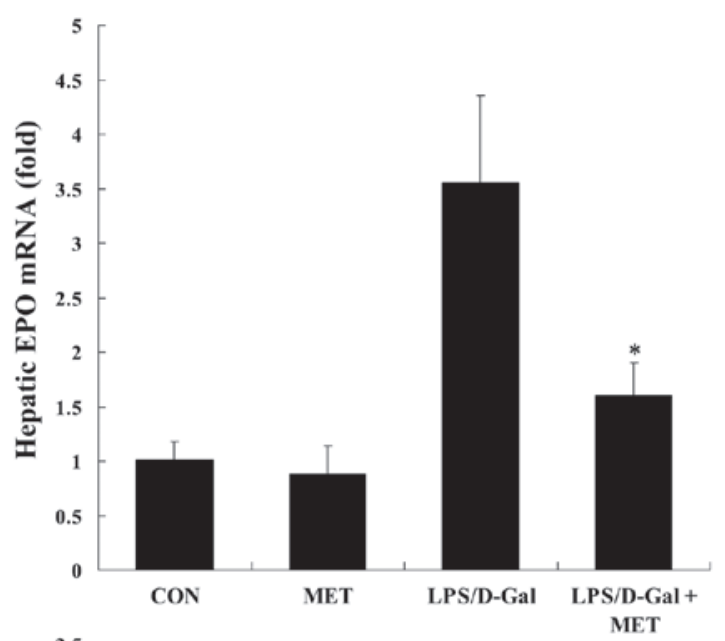

B

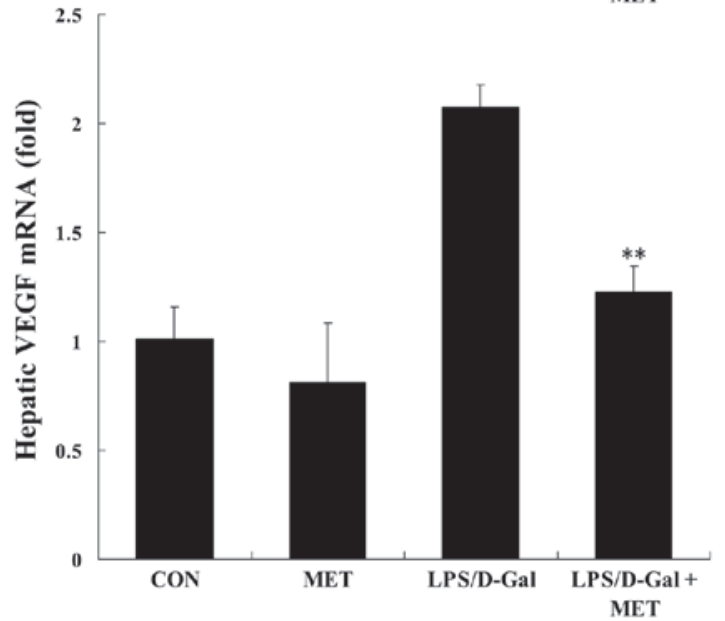

C

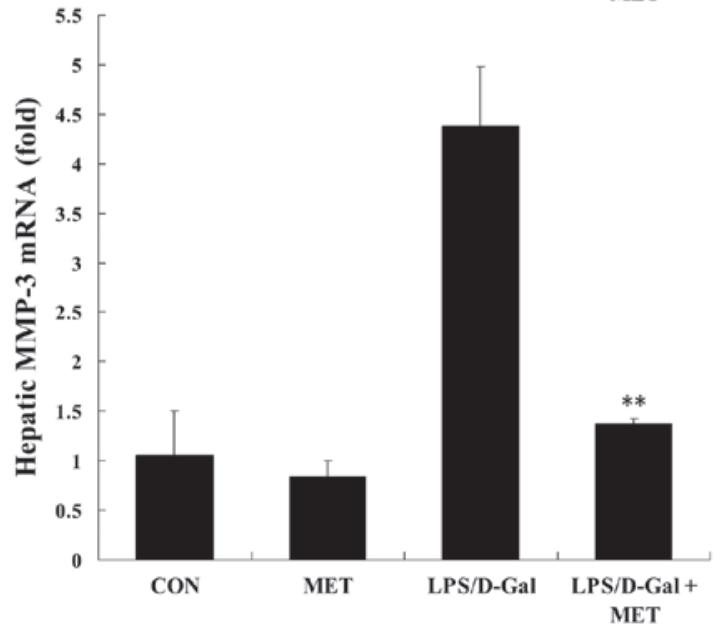

Figure 6. MET suppressed LPS/D-Gal-induced upregulation of EPO, VEGF and MMP-3. Mice were treated with the vehicle or MET $(400 \mathrm{mg} / \mathrm{kg})$ in the presence or absence of LPS/D-Gal exposure. Liver samples were harvested $6 \mathrm{~h}$ subsequent to LPS/D-Gal exposure and the mRNA levels of (A) EPO, (B) VEGF and (C) MMP-3 were determined. Data are presented as the mean \pm standard deviation, $\mathrm{n}=8 .{ }^{*} \mathrm{P}<0.05,{ }^{* *} \mathrm{P}<0.01$ vs. LPS/D-Gal group. MET, metformin; LPS, lipopolysaccharide; D-Gal, D-galactosamine; EPO, erythropoietin; VEGF, vascular endothelial growth factor; MMP-3, matrix metalloproteinase-3; CON, control.

effects of metformin on the plasma protein levels of IL-6 in LPS/D-Gal-exposed mice were observed ( $\mathrm{P}<0.05$; Fig. 3B).

Metformin suppresses LPS/D-Gal-induced NF- $\kappa B$ translocation. NF- $\mathrm{KB}$ is a pivotal transcriptional factor involved in the

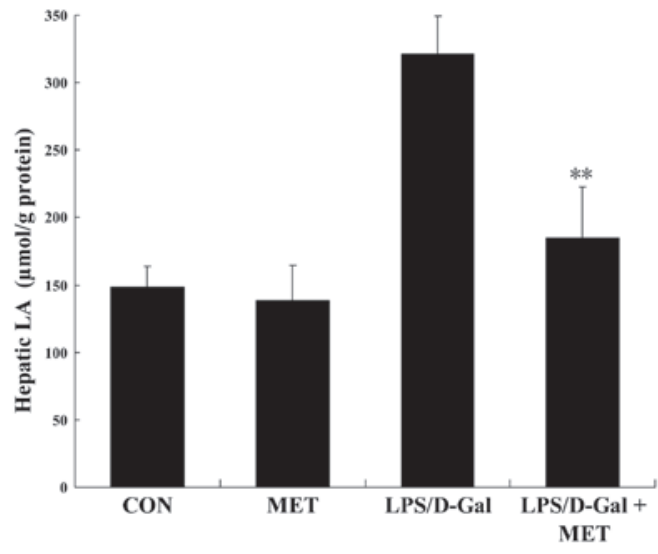

Figure 7. MET suppressed LPS/D-Gal-induced upregulation of LA. Mice were treated with the vehicle or MET $(400 \mathrm{mg} / \mathrm{kg})$ in the presence or absence of LPS/D-Gal exposure. Liver samples were harvested $6 \mathrm{~h}$ subsequent to LPS/D-Gal exposure and the hepatic levels of LA were determined. Data are presented as the mean \pm standard deviation, $n=8 .{ }^{* *} \mathrm{P}<0.01 \mathrm{vs}$. LPS/D-Gal group. MET, metformin; LPS, lipopolysaccharide; D-Gal, D-galactosamine; LA, lactic acid; CON, control.

transcriptional regulation of IL- 6 and TF, and the nuclear translocation of NF- $\kappa \mathrm{B}$ is crucial for its activation $(23,24)$. In the present study, the exposure to LPS/D-Gal markedly induced the translocation of NF- $\mathrm{KB}$ p 65 into the nuclei indicated by the increased nuclear level of $\mathrm{p} 65$ following LPS/D-Gal challenge, whilst treatment with metformin significantly reduced the nuclear level of $\mathrm{p} 65$ ( $\mathrm{P}=0.023$; Fig. 4).

Metformin alleviates LPS/D-Gal-induced hypoxia. Tissue hypoperfusion and hypoxia occur as a consequence of coagulation activation (12). In the present study, the degree of intrahepatic hypoxia was evaluated via the detection of the level of HIF-1 $\alpha$. These data demonstrate that metformin significantly suppressed the upregulation of HIF-1 $\alpha$ protein levels ( $\mathrm{P}<0.01$; Fig. 5). In agreement with this, HIF-1-targeted genes, including EPO, VEGF and MMP-3 $(25,26)$, were upregulated following LPS/D-Gal exposure, however this upregulation was suppressed by metformin (Fig. 6).

Metformin alleviates LPS/D-Gal-induced metabolic disturbances. The metabolic disturbance resulting from hypoxia was determined by detecting the level of LA, an anaerobic metabolic product. The data collected indicate that LPS/D-Gal exposure significantly increased the hepatic level of LA, which was inhibited by metformin (Fig. 7).

\section{Discussion}

Metformin is used in the clinical treatment of diabetes due to its hypoglycemic activity (27). In addition, studies have identified the anti-inflammatory properties of metformin in vitro and in vivo. In an LPS/D-Gal-induced hepatitis mouse model (28-30), it was demonstrated that treatment with the antidiabetic drug metformin provided therapeutic benefits. This suggested that these protective effects may result from the reduced production of the pro-inflammatory cytokine, TNF- $\alpha$ (18), a major detrimental and proapoptotic factor in this model of hepatitis (19). In the present study, it 
was demonstrated that metformin significantly suppressed LPS/D-Gal-induced coagulation activation, as indicated by the reduced expression levels of TF and PAI-1, reduced level of HIF-1 and reduced content of LA in the liver.

The induction of TF serves a role in the activation of coagulation under inflammatory circumstances (8). TF is constitutively expressed in the majority of tissues, however is not expressed in cells in direct contact with the blood (14). Once the integrity of a vessel wall is disrupted, TF comes into contact with other coagulation factors in the blood and initiates coagulation (14). Additionally, the expression of TF may be induced by inflammatory stimuli. Therefore, the cells in direct contact with the blood, such as monocytes and endothelial cells, may be induced to express TF under inflammatory circumstances (13). Several pro-inflammatory cytokines have been suggested to mediate the induction of TF expression, however IL- 6 may be the most important mediator, as inhibition of IL- 6 reverses TF-dependent thrombin generation in experimental endotoxemia $(22,31)$. In the present study, the level of IL- 6 and TF increased significantly in mice challenged with LPS/D-Gal, however this upregulation was suppressed by metformin, suggesting that the key steps in the activation of the coagulation system were inhibited by metformin.

The formation of thrombin may be counter-regulated by the plasmin-catalyzed fibrinolysis process (32). Plasmin is generated from its precursor plasminogen and this conversion is stimulated by two activators, urokinase-type and tissue-type plasminogen activator (32), which are inhibited by PAI-1 (33). Additionally, PAI-1 is associated with inflammation due to the previously reported potential upregulation of expression of PAI-1 by inflammatory stimuli (34). In the present study, the LPS/D-Gal-induced expression of PAI-1 was significantly reduced by metformin, suggesting that the suppression of fibrinolysis may be reversed by metformin. Previous studies have demonstrated that TNF- $\alpha$ and IL- 6 contribute to the increased expression of PAI-1 under inflammatory conditions (34). In addition, the induced production of TNF- $\alpha$ has been demonstrated to be significantly inhibited by metformin (18), with the present study indicating that the expression of IL-6 was also suppressed. These anti-inflammatory effects of metformin may result in reduced expression of PAI-1.

The transcription factor $\mathrm{NF}-\kappa \mathrm{B}$ controls the expression of numerous pro-inflammatory genes including TNF- $\alpha$ and IL-6 (35). In unstimulated cells, $N F-\kappa B$ is sequestered by its inhibitor, inhibitory $\kappa \mathrm{B}(\mathrm{I} \kappa \mathrm{B})(36)$. Inflammatory stimuli may induce the degradation of $\mathrm{I} \kappa \mathrm{B}$ and the release of $\mathrm{NF}-\kappa \mathrm{B}$, resulting in the translocation of $\mathrm{NF}-\kappa \mathrm{B}$ into the nucleus, where it activates the transcription of target genes (36). In the present study, the increased level of nuclear $\mathrm{NF}-\kappa \mathrm{B}$ in LPS/D-Gal-challenged mice was partially reversed by metformin, which may contribute to the anti-inflammatory effect on TNF- $\alpha$ and IL- 6 expression. Additionally, there is evidence indicating that $\mathrm{NF}-\kappa \mathrm{B}$ is involved in the transcriptional regulation of TF and PAI-1 $(24,34)$. Thus, the inhibitory effects on NF- $\kappa$ B activation may underlie the reduced induction of TF and PAI-1.

The activation of the coagulation system may result in the reduction of blood flow, which leads to tissue hypoperfusion and hypoxia (12). In the present study, the degree of hepatic hypoxia was determined via the detection of the stable protein level of HIF-1 $\alpha$. HIF is an oxygen-sensitive transcription factor containing an oxygen-labile $\alpha$-subunit and a constitutively expressed $\beta$-subunit (37). Under normoxic conditions, the hydroxylation of HIF-1 $\alpha$ by the prolyl-4-hydroxylase domain-containing enzymes (PHD) targets HIF-1 $\alpha$ for polyubiquitination and proteosomal degradation (38). In hypoxic conditions, PHD activity is inhibited and HIF-1 $\alpha$ accumulates and binds to the hypoxia-responsive element sequences of target gene promoters (38). In mice exposed to LPS/D-Gal, levels of the stable protein of HIF-1 $\alpha$ were markedly increased, suggesting the presence of severe hypoxia in the liver tissues. Treatment with metformin significantly reduced the level of HIF-1 $\alpha$ protein in LPS/D-Gal-challenged mice. Additionally, the LPS/D-Gal-induced expression of HIF-1-targeted genes, including EPO, VEGF and MMP-3 (25-26), was suppressed following metformin treatment. These data were consistent with the reduced level of TF. The HIF-1-driven expression of harmful products such as MMP-3 may represent one of the mechanisms involved in hypoxia-induced tissue damage (39).

Furthermore, tissue hypoxia and hypoperfusion may lead to metabolic disturbance. Hypoxia may block mitochondrial oxidative phosphorylation resulting in the generation of ATP from the anaerobic metabolic pathway. In the anaerobic metabolic pathway, pyruvate is converted into LA instead of acetyl-coenzyme A (40). In the present study, the level of LA increased significantly following LPS/D-Gal exposure, however treatment with metformin reduced the level of LA. Increased levels of LA have been suggested to be a pathological factor responsible for liver injury via the stimulation of the release of hydrolytic lysosomal enzymes into the cytosol and other mechanisms, including the induction of endothelial dysfunction $(41,42)$.

Taken together, the results of the present study further indicate that metformin significantly suppresses LPS/D-Gal-induced NF- $\kappa$ B activation, and IL-6, TF and PAI-1 expression. These effects are suggested to be associated with alleviated hepatic hypoxia and metabolic disturbance. Therefore, the present study identified an additional mechanism underlying the hepatoprotective effects of metformin in mice with LPS/D-Gal-induced fulminant hepatitis. The suppressive effects of metformin on inflammation and inflammation-induced coagulation may contribute to the protective benefits of metformin.

\section{Acknowledgements}

The current study was supported by a grant from the National Natural Science Foundation of China (grant no. 81370179) and a grant from Chongqing Municipal Education Commission (grant no. KJ1400235).

\section{References}

1. Schiødt FV and Lee WM: Fulminant liver disease. Clin Liver Dis 7: 331-349, vi, 2003.

2. Bernal W, Auzinger G, Dhawan A and Wendon J: Acute liver failure. Lancet 376: 190-201, 2010.

3. Tsutsui H, Adachi K, Seki E and Nakanishi K: Cytokine-induced inflammatory liver injuries. Curr Mol Med 3: 545-559, 2003.

4. Hatano E: Tumor necrosis factor signaling in hepatocyte apoptosis. J Gastroenterol Hepatol 22 (Suppl 1): S43-S44, 2007. 
5. Kim SJ and Lee SM: NLRP3 inflammasome activation in D-galactosamine and lipopolysaccharide-induced acute liver failure: Role of heme oxygenase-1. Free Radic Biol Med 65: 997-1004, 2013.

6. Li Y, Xu Z, Yu Y, Yuan H, Xu H, Zhu Q, Wang C and Shi X: The vagus nerve attenuates fulminant hepatitis by activating the Src kinase in Kuppfer cells. Scand J Immunol 79: 105-112, 2014

7. Levi M, Schultz M and van der Poll T: Sepsis and thrombosis. Semin Thromb Hemost 39: 559-566, 2013.

8. Aksu K, Donmez A and Keser G: Inflammation-induced thrombosis: Mechanisms, disease associations and management. Curr Pharm Des 18: 1478-1493, 2012.

9. Semeraro N, Ammollo CT, Semeraro F and Colucci M: Sepsis, thrombosis and organ dysfunction. Thromb Res 129: 290-295, 2012.

10. O'Brien M: The reciprocal relationship between inflammation and coagulation. Top Companion Anim Med 27: 46-52, 2012.

11. de Laforcade A: Diseases associated with thrombosis. Top Companion Anim Med 27: 59-64, 2012

12. Gando S: Microvascular thrombosis and multiple organ dysfunction syndrome. Crit Care Med 38 (Suppl): S35-S42, 2010.

13. Egorina EM, Sovershaev MA and Hansen JB: The role of tissue factor in systemic inflammatory response syndrome. Blood Coagul Fibrinolysis 22: 451-456, 2011.

14. Mackman N: The many faces of tissue factor. J Thromb Haemost 7 (Suppl 1): 136-139, 2009.

15. Schouten M, Wiersinga WJ, Levi $M$ and van der Poll $T$ : Inflammation, endothelium, and coagulation in sepsis. J Leukoc Biol 83: 536-545, 2008

16. Bergmann S and Hammerschmidt S: Fibrinolysis and host response in bacterial infections. Thromb Haemost 98: 512-520, 2007.

17. Hermans PW and Hazelzet JA: Plasminogen activator inhibitor type 1 gene polymorphism and sepsis. Clin Infect Dis 41 (Suppl 7): S453-S458, 2005.

18. Yuan H, Li L, Zheng W, Wan J, Ge P, Li H and Zhang L: Antidiabetic drug metformin alleviates endotoxin-induced fulminant liver injury in mice. Int Immunopharmacol 12 : 682-688, 2012

19. Silverstein R: D-galactosamine lethality model: Scope and limitations. J Endotoxin Res 10: 147-162, 2004.

20. Miyazaki M, Kato M, Tanaka M, Tanaka K, Takao S, Kohjima M, Ito T, Enjoji M, Nakamuta M, Kotoh K, et al: Antithrombin III injection via the portal vein suppresses liver damage. World J Gastroenterol 18: 1884-1891, 2012

21. Takano H, Inoue K, Shimada A, Sato H, Yanagisawa R and Yoshikawa T: Urinary trypsin inhibitor protects against liver injury and coagulation pathway dysregulation induced by lipopolysaccharide/D-galactosamine in mice. Lab Invest 89: 833-839, 2009

22. Kerr R, Stirling D and Ludlam CA: Interleukin 6 and haemostasis. Br J Haematol 115: 3-12, 2001.

23. Liang DY, Liu LM, Ye CG, Zhao L, Yu FP, Gao DY, Wang YY, Yang ZW and Wang YY: Inhibition of UII/UTR system relieves acute inflammation of liver through preventing activation of NF- $\kappa$ B pathway in ALF mice. PLoS One 8: e64895, 2014.

24. Jiang R, Wang NP, Tanaka KA, Levy JH, Guyton RA, Zhao ZQ and Vinten-Johansen J: Factor Xa induces tissue factor expression in endothelial cells by P44/42 MAPK and NF- $\kappa \mathrm{B}$-dependent pathways. J Surg Res 169: 319-327, 2011.
25. Rhim T, Lee DY and Lee M: Hypoxia as a target for tissue specific gene therapy. J Control Release 172: 484-494, 2013.

26. Ahn JK, Koh EM, Cha HS, Lee YS, Kim J, Bae EK and Ahn KS: Role of hypoxia-inducible factor-1alpha in hypoxia-induced expressions of IL-8, MMP-1 and MMP-3 in rheumatoid fibroblast-like synoviocytes. Rheumatology (Oxford) 47: 834-839, 2008

27. Bailey CJ and Turner RC: Metformin. N Engl J Med 334 574-579, 1996.

28. Tsoyi K, Jang HJ, Nizamutdinova IT, Kim YM, Lee YS, Kim HJ, Seo HG, Lee JH and Chang KC: Metformin inhibits HMGB1 release in LPS-treated RAW 264.7 cells and increases survival rate of endotoxaemic mice. Br J Pharmacol 162: $1498-1508,2011$

29. Hattori Y, Suzuki K, Hattori S and Kasai K: Metformin inhibits cytokine-induced nuclear factor kappaB activation via AMP-activated protein kinase activation in vascular endothelial cells. Hypertension 47: 1183-1188, 2006.

30. Nath N, Khan M, Paintlia MK, Singh I, Hoda MN and Giri S: Metformin attenuated the autoimmune disease of the central nervous system in animal models of multiple sclerosis. J Immunol 182: 8005-8014, 2009.

31. van der Poll T, Levi M, Hack CE, ten Cate H, van Deventer SJ, Eerenberg AJ, de Groot ER, Jansen J, Gallati H, Büller HR, et al: Elimination of interleukin 6 attenuates coagulation activation in experimental endotoxemia in chimpanzees. J Exp Med 179: 1253-1259, 1994

32. Wyseure T and Declerck PJ: Novel or expanding current targets in fibrinolysis. Drug Discov Today 19: 1476-1482, 2014.

33. Dupont DM, Madsen JB, Kristensen T, Bodker JS, Blouse GE, Wind T and Andreasen PA: Biochemical properties of plasminogen activator inhibitor-1. Front Biosci (Landmark Ed) 14: 1337-1361, 2009

34. Kruithof EK: Regulation of plasminogen activator inhibitor type 1 gene expression by inflammatory mediators and statins. Thromb Haemost 100: 969-975, 2008.

35 . Hoesel B and Schmid JA: The complexity of NF- $\kappa \mathrm{B}$ signaling in inflammation and cancer. Mol Cancer 12: 86, 2013.

36. Lee IT and Yang CM: Inflammatory signalings involved in airway and pulmonary diseases. Mediators Inflamm 2013 791231, 2013

37. Ong SG and Hausenloy DJ: Hypoxia-inducible factor as a therapeutic target for cardioprotection. Pharmacol Ther 136: 69-81, 2012.

38. Brocato J, Chervona Y and Costa M: Molecular responses to hypoxia-inducible factor $1 \alpha$ and beyond. Mol Pharmacol 85: 651-657, 2014.

39. Ramakrishnan P, Hecht BA, Pedersen DR, Lavery MR, Maynard J, Buckwalter JA and Martin JA: Oxidant conditioning protects cartilage from mechanically induced damage. J Orthop Res 28: 914-920, 2010

40. Levy B: Lactate and shock state: The metabolic view. Curr Opin Crit Care 12: 315-321, 2006.

41. Soric S, Belanger MP, Askin N and Wittnich C: Impact of female sex hormones on liver tissue lactic acidosis during ischemia. Transplantation 84: 763-770, 2007.

42. James JH, Luchette FA, McCarter FD and Fischer JE: Lactate is an unreliable indicator of tissue hypoxia in injury or sepsis. Lancet 354: 505-508, 1999. 